\title{
Changes in oocyte plasma membrane binding sites on boar spermatozoa with capacitation and acrosome reactions
}

\author{
B. L. Sartini* and T. Berger ${ }^{\dagger}$ \\ Department of Animal Science, University of California, Davis, CA 95616, USA
}

\begin{abstract}
The objective of this study was to determine the localization and distribution of oocyte plasma membrane binding sites on capacitated and acrosome-reacting live boar spermatozoa. Localization of oocyte plasma membrane binding sites on boar spermatozoa was determined with fluorescence microscopy and population distribution was examined with flow cytometry. The number of spermatozoa with oocyte plasma membrane bound to the equatorial segment and postacrosomal region of the sperm head significantly increased with capacitation. Equatorial segment labelling further increased with induced acrosome reactions. When the population distribution of oocyte
\end{abstract}

plasma membrane binding sites on live boar spermatozoa was analysed, the percentage of spermatozoa with bound oocyte plasma membrane significantly increased after capacitation compared with that of washed spermatozoa. Binding of oocyte plasma membrane did not increase in control spermatozoa incubated under non-capacitating conditions and was not correlated with the percentage of dead spermatozoa. A change in localization of oocyte plasma membrane binding sites on the sperm head was demonstrated using fluorescence microscopy and an increase in oocyte plasma membrane binding sites after capacitation was shown using flow cytometry.

\section{Introduction}

During mammalian fertilization, sperm and oocyte membrane interaction leads to integration of the two membranes enclosing a single diploid embryo. In preparation for fusion with the oocyte plasma membrane, the capacitated spermatozoon undergoes an acrosome reaction leading to loss of the plasma membrane over the anterior head of the spermatozoon and exposing the inner acrosomal membrane. Plasma membrane remains over the equatorial segment, postacrosomal head, midpiece and tail of the spermatozoon after the acrosome reaction. Morphological studies using rodent species have demonstrated that molecules on the exposed inner acrosomal membrane initially bind to the oocyte plasma membrane (Koehler et al., 1982; Talbot and Chacon, 1982). Fusion of the sperm and oocyte membranes occurs at the equatorial segment of the sperm head and incorporates the postacrosomal head plasma membrane (Yanagimachi, 1994).

Modification of the sperm plasma membrane occurs during capacitation and the acrosome reaction to expose oocyte interaction proteins on the sperm surface. These modifications include unmasking sites and movement of sites to the correct location on the sperm head in

*Present address: Department of Animal Science, Morrison Hall, Cornell University, Ithaca, NY 14850, USA

${ }^{\dagger}$ Correspondence

Email: tberger@ucdavis.edu preparation for fusion with the oocyte plasma membrane (de Lamirande et al., 1997). Migration of individual sperm proteins, including potential sperm ligands for the oocyte, between sperm surface domains to the equatorial and postacrosomal regions has been demonstrated in some rodent species (Primakoff et al., 1987; Rochwerger and Cuasnicu, 1992). Relocation of oocyte ligands on the boar sperm plasma membrane has not been investigated.

The aim of the present study was to determine the localization of oocyte binding sites on individual boar sperm cells and the population distribution of oocyte plasma membrane binding sites in boar sperm populations during capacitation and acrosome reaction. Owing to its ability to analyse thousands of spermatozoa and provide quantitative comparisons in oocyte plasma membrane binding with changes in functional status, flow cytometric analysis was used to determine the distribution of oocyte plasma membrane binding sites in boar sperm populations.

\section{Materials and Methods}

\section{Sperm collection and processing}

Sperm-rich fractions were collected from boar (PIC L27 $\times$ L15, PIC L27 × 1050 and Yorkshire) semen samples, filtered through Miracloth, assessed for motility and washed on a Percoll gradient in Tris-buffered medium (TBM; $113.1 \mathrm{mmol} \mathrm{NaCl} \mathrm{I}{ }^{-1}, 10 \mathrm{mmol} \mathrm{CaCl}_{2} \mathrm{I}^{-1}$, $20 \mathrm{mmol}$ Tris $\mathrm{I}^{-1}, 10 \mathrm{mmol}$ glucose $\mathrm{I}^{-1}, 0.5 \mathrm{mmol}$ 
sodium pyruvate $\mathrm{I}^{-1}, 3 \mathrm{mmol} \mathrm{KCl} \mathrm{I}^{-1}, 50 \mu \mathrm{g}$ gentamicin sulphate $\left.\mathrm{ml}^{-1}, \mathrm{pH} 7.85\right)$ as described by Berger and Horton (1988). Spermatozoa were diluted to $1 \times 10^{7}$ spermatozoa $\mathrm{ml}^{-1}$ in $5 \mathrm{mg} \mathrm{BSA} \mathrm{ml} \mathrm{m}^{-1}$ of TBM (washed spermatozoa). For capacitation, an aliquot of these washed spermatozoa was incubated at $39^{\circ} \mathrm{C}$ with $5 \%$ $\mathrm{CO}_{2}$ in air for $6 \mathrm{~h}$ (capacitated spermatozoa). Acrosome reactions were induced by addition of $125 \mu \mathrm{g}$ heatsolubilized pig zona pellucida $\mathrm{ml}^{-1}$ for $30 \mathrm{~min}$ after $5.5 \mathrm{~h}$ of incubation (Berger et al., 1989). As a control for capacitation (control incubated spermatozoa), an aliquot of the sperm-rich fraction was washed on a Percoll gradient and incubated with $5 \mathrm{mg}$ polyvinyl alcohol (PVA) $\mathrm{ml}^{-1}$ of TBM instead of BSA at $39^{\circ} \mathrm{C}$ for $6 \mathrm{~h}$ in the absence of $\mathrm{CO}_{2}$.

\section{Isolation and biotinylation of pig oocyte plasma membrane}

Oocyte plasma membrane was isolated and biotinylated as described by Ash et al. (1994). Briefly, pig ovaries were sliced and cumulus-free oocytes with intact zona pellucida were isolated by filtration. After homogenization and removal of the zona pellucida, oocyte plasma membrane was isolated by differential centrifugation. The protein content of the isolated oocyte plasma membrane was determined with a bicinchoninic acid assay (Pierce, Rockford, IL). Isolated pig oocyte plasma membrane was solubilized in $16 \mathrm{mmol} 3$-[(3-cholamidopropyl)dimethylammonio]1-propanesulphonate (Chaps) I-1, 20\% (v/v) glycerol, $2 \mathrm{mmol}$ EDTA I ${ }^{-1}$ and $10 \mathrm{mmol} \mathrm{NaHCO}_{3} \mathrm{I}^{-1}$ buffer at $4^{\circ} \mathrm{C}$ for $1 \mathrm{~h}$ with gentle shaking before biotinylation. Biotin label (NHS-LC-Biotin, Pierce) was added to solubilized oocyte plasma membrane at a ratio of 2:1 (w:w) biotin:oocyte plasma membrane. Biotinylation occurred for $2 \mathrm{~h}$ at $4^{\circ} \mathrm{C}$ with gentle shaking. Unreacted biotin was removed by ultrafiltration with $10 \mathrm{mmol} \mathrm{NaHCO}_{3} \mathrm{I}^{-1}$ in Centricon ultrafiltration devices (Amicon, Beverley, MA). Biotinylated oocyte plasma membrane was stored at $-20^{\circ} \mathrm{C}$ until used.

\section{Indirect fluorescent labelling of live boar spermatozoa}

Live washed, capacitated and zona pellucida-induced acrosome-reacting spermatozoa $\left(1 \times 10^{7}\right.$ spermatozoa $\mathrm{ml}^{-1}$ TBM) from three boars were incubated with biotinylated oocyte plasma membrane $\left(20 \mu \mathrm{g} \mathrm{m}^{-1}\right)$ for $10 \mathrm{~min}$. Unbound oocyte plasma membrane was removed by centrifugation at $350 \mathrm{~g}$ for $3 \mathrm{~min}$ and the supernatant was discarded. Alexa Fluor ${ }^{\circledR}$ 488-avidin (Alexa 488-avidin, Molecular Probes, Eugene, OR) was added at $2 \mu \mathrm{g} \mu \mathrm{I}^{-1}$ in TBM to the sperm pellet and incubated for $10 \mathrm{~min}$. Sperm aliquots were centrifuged at $350 \mathrm{~g}$ for $3 \mathrm{~min}$ to remove unbound excess Alexa 488avidin and spermatozoa were resuspended in TBM. As a negative control, only the Alexa 488-avidin was added to sperm aliquots (no biotinylated oocyte plasma membrane was added). The pattern of bound oocyte plasma membrane was visualized with fluorescence microscopy and scored for 300 spermatozoa per treatment. The percentage of spermatozoa with different oocyte plasma membrane staining patterns was analysed using the general linear models (GLM) procedure in SAS (SAS Institute Inc., 1985). Differences among means were tested with linear contrasts.

\section{Flow cytometry of oocyte plasma membrane-labelled live boar spermatozoa}

Live boar spermatozoa were incubated with Alexa 488-avidin-labelled biotinylated oocyte plasma membrane to detect oocyte binding sites by flow cytometry analysis. Biotinylated oocyte plasma membrane was directly labelled with a 2:1 ratio (w:w) of Alexa 488avidin (OPM-Alexa). For a negative control, a 2:1 ratio (w:w) of Alexa 488-avidin was added to unbiotinylated oocyte plasma membrane (unbiotinylated OPM-Alexa). In preliminary experiments, the removal of excess Alexa 488-avidin was unnecessary as it did not interfere with detection of the bound OPM-Alexa. Washed, capacitated, control-incubated, and zona pellucida-induced acrosome-reacting spermatozoa $\left(1 \times 10^{7}\right.$ spermatozoa $\mathrm{ml}^{-1}$ TBM) from each of the three different boars were labelled with $0.002 \mu \mathrm{g}$ OPM-Alexa $\mu \mathrm{I}^{-1}$ or $0.002 \mu \mathrm{g}$ unbiotinylated OPM-Alexa $\mu \mathrm{I}^{-1}$ in TBM for $5 \mathrm{~min}$. OPM-Alexa-bound boar sperm samples were diluted fivefold in TBM to $2 \times 10^{6}$ spermatozoa $\mathrm{ml}^{-1}$ for flow cytometry analysis. Propidium iodide (PI) was added to OPM-Alexa-labelled boar spermatozoa to a final concentration of $12 \mu \mathrm{mol} \mathrm{I}^{-1}$ to discriminate between live and dead spermatozoa in treatment samples.

Washed, capacitated, control incubated, and zona pellucida-induced acrosome-reacting boar sperm aliquots that were incubated with OPM-Alexa and unbiotinylated OPM-Alexa were analysed by FACScan (Becton-Dickinson, San Jose, CA). The FACScan was equipped with a 488 argon laser, FL1 530 bandpass filter and FL3 $620 \mathrm{~nm}$ longpass filters. Data were collected for forward scatter (FSC), side scatter (SSC), FL1 (OPM Alexa 488-avidin) and FL3 (PI). Data from a minimum of 10000 spermatozoa were collected per treatment.

\section{Analysis of flow cytometry data}

Data were analysed using the WinMIDI flow cytometry program (version 3.0, J. Trotter, free software). Initially, regional gates were set for FSC and SSC on PI-stained dead sperm samples to identify the sperm population in live sperm samples and remove small particles and sperm aggregates from analysis. The distribution of OPM-Alexa-labelled boar spermatozoa and unbiotinylated OPM-Alexa negative control sperm populations were compared using the Kolmogorov-Smirnov (K-S) statistic (Young, 1977). The 
$\mathrm{K}-\mathrm{S}$ statistic is used in flow cytometric analysis to determine whether two population distributions are from the same parent distribution. Significance indicates that the distributions being compared are not from the same parent distribution.

The labelling frequency of the OPM-Alexa-bound sperm population was determined by visually assessing the distributions of the treatment population (OPM-Alexa) with the control population (unbiotinylated OPM-Alexa) on the same histogram. The lowest channel number in which the OPM-Alexa binding was higher than the control binding was determined. The percentage of the OPM-Alexa and control sperm populations over that channel number was determined and the percentage of spermatozoa in the control population was subtracted from the percentage of the OPM-Alexa-bound population. Labelling frequency of treatments and correlation of live and dead sperm populations with percentage of OPM-Alexa-labelled population were analysed using the GLM procedure in SAS (SAS Institute Inc., 1985). Differences among means were tested with linear contrasts.

\section{Results}

Oocyte plasma membrane fluorescent labelling patterns on live boar spermatozoa

Localization of oocyte plasma membrane on the head of live boar spermatozoa changed with capacitation and (a)

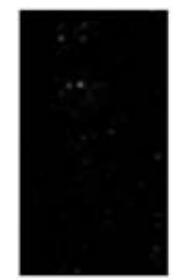

(b)
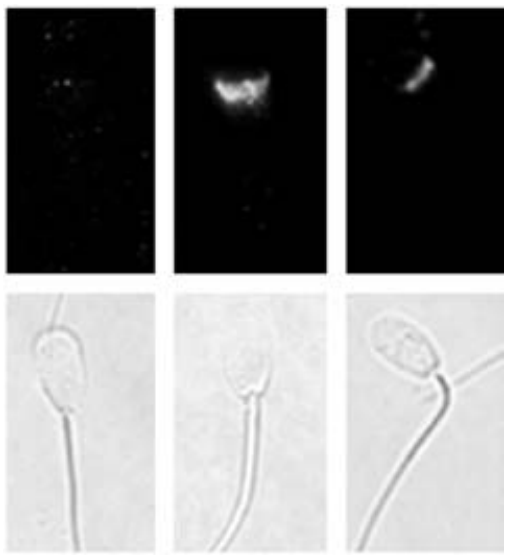

Fig. 1. Labelling of unfixed boar spermatozoa with biotinylated oocyte plasma membrane. (a) Washed spermatozoa, (b) capacitated spermatozoa and (c) capacitated spermatozoa after addition of solubilized zona pellucida to induce acrosome reactions.

induced acrosome reactions. In the washed boar sperm population, oocyte plasma membrane bound to $2.2 \%$ of the sperm population (whole head and faint head) while $97.8 \%$ of the population remained unbound by the oocyte plasma membrane as detected by fluorescent microscopy (Figs 1 and 2). Postacrosomal head and equatorial segment localization of the bound oocyte plasma membrane occurred when boar spermatozoa

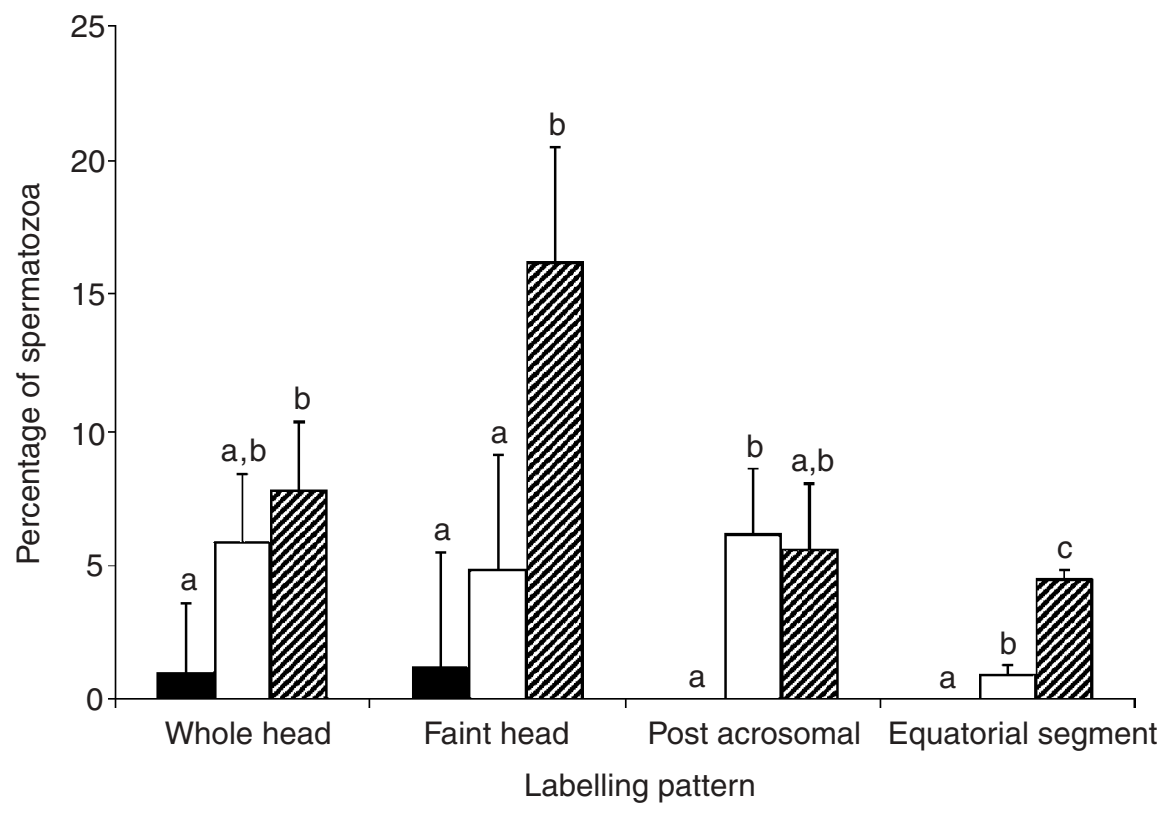

Fig. 2. Binding patterns of biotinylated oocyte plasma membrane on washed capacitated ( $\square$ ) and zona pellucida-induced, acrosome-reacting boar spermatozoa (婮). Control staining was negligible. ${ }^{a, b}$ Values with no letters in common are significantly different among sperm populations, $P<0.05$. ' $V a l u e s$ are significantly different from populations designated with an ${ }^{\mathrm{a}}$ or ${ }^{\mathrm{b}}, P<0.0001$. 


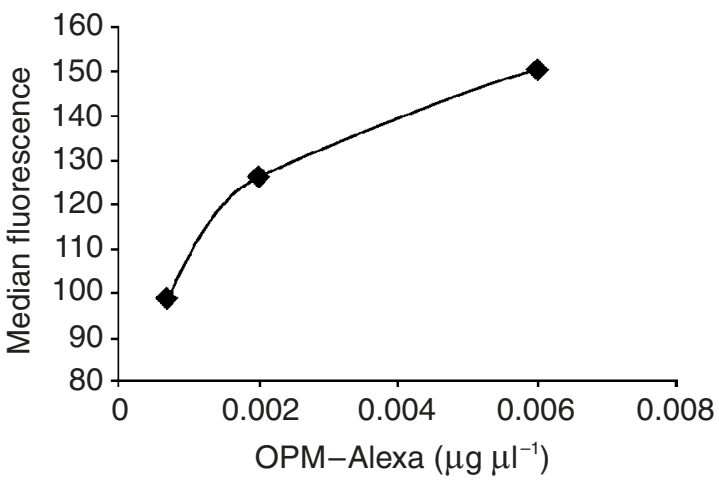

Fig. 3. Concentration dependence of OPM-Alexa-labelled oocyte binding sites on boar spermatozoa with flow cytometry. Median fluorescence: channel number from cytometric analysis for median fluorescent intensity of population.

were incubated under capacitating conditions for $6 \mathrm{~h}$. After induction of acrosome reactions with solubilized zona pellucida, the percentage of spermatozoa with equatorial localization of the bound oocyte plasma membrane significantly increased compared with the percentage at capacitation (4.5 versus 1.0, SEM $=0.4$, $P<0.0001, n=3)$. The percentage of spermatozoa with faint head labelling with the oocyte plasma membrane was significantly increased in the acrosome-reacting population when compared with that of washed spermatozoa or capacitated spermatozoa $(16.2 \%$ versus $1.2 \%$ or $4.8 \%$ for acrosome-reacting, washed or capacitated spermatozoa, respectively, SEM $=4.3, P<0.05)$.

Flow cytometric detection of oocyte plasma membrane binding sites in boar sperm populations

Three concentrations of OPM-Alexa were initially incubated with spermatozoa to determine an optimal concentration for flow cytometric analysis. When OPMAlexa binding site data were analysed on a histogram, a concentration-dependent change in median channel number occurred with increasing concentrations of OPM-Alexa (Fig. 3). The concentration of $0.002 \mu \mathrm{g}$ OPM-Alexa $\mu \mathrm{I}^{-1}$ was chosen for subsequent experiments. Four sperm populations were labelled with OPM-Alexa for each of three boars: washed, capacitated, incubated control and zona pellucidainduced acrosome-reacting capacitated spermatozoa. The distribution was significantly different from the negative control population (unbiotinylated OPM-Alexa) for each population from each boar as indicated by $\mathrm{K}-\mathrm{S}$ statistical tests $(P<0.001$; data not shown).

The subpopulation of spermatozoa that bound oocyte plasma membrane increased from $21.0 \%$ of washed spermatozoa to $38.2 \%$ of capacitated spermatozoa $(P=0.005$, SEM $=4.0, n=3$; Fig. 4$)$. As an incubation control for capacitation, spermatozoa incubated with PVA instead of BSA and in the absence of bicarbonate were incubated with OPM-Alexa. The increase in

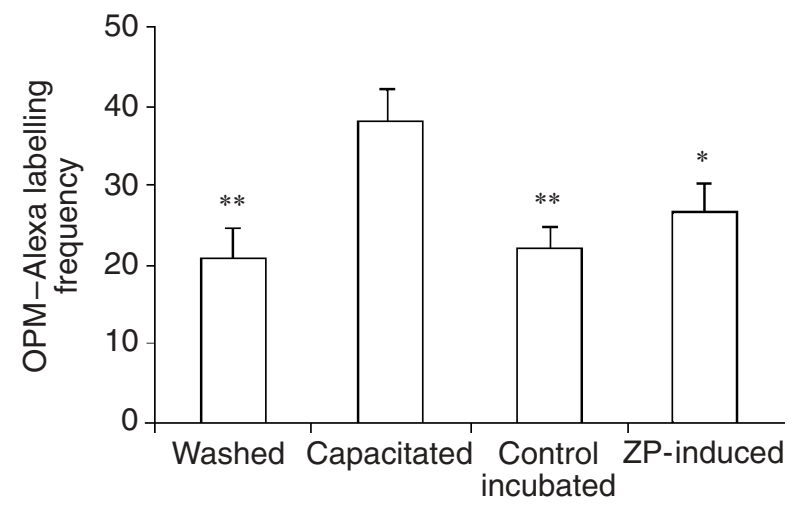

Fig. 4. Labelling frequency of boar spermatozoa with OPMAlexa as determined by flow cytometry. Data represent percentage of spermatozoa positively labelled with OPM-Alexa after subtraction of percentage of spermatozoa labelled in negative controls (unbiotinylated OPM + Alexa avidin). ZP: zona pellucida. *Significantly different from capacitated sperm populations, $P<$ 0.05 . ${ }^{*}$ Significantly different from capacitated sperm populations, $P<0.01$

OPM-Alexa binding to spermatozoa that occurred after incubation under capacitating conditions was not detected in the spermatozoa incubated with PVA without bicarbonate $(22.2 \%$ of the population; $P=0.007 \mathrm{com}$ pared with capacitated spermatozoa). The percentage of spermatozoa binding OPM-Alexa in the zona pellucida induced acrosome-reacting population $(26.7 \%)$ was less than the percentage detected in the capacitated sperm treatment $(P=0.03)$.

Median fluorescence was similar in washed $(\mu=137)$, capacitated $(\mu=143)$ and zona pellucida-induced acrosome-reacting populations $(\mu=136)$. Although more capacitated spermatozoa bound OPM-Alexa, capacitation did not increase the amount bound by the average individual responding spermatozoon in these experiments.

Dead spermatozoa were clearly separated from live spermatozoa using $\mathrm{PI}$ in conjunction with OPMAlexa staining (Fig. 5). The percentages of neither live $(62.8 \%)$ nor dead spermatozoa (36\%) were significantly correlated with the percentage of OPM-Alexa-labelled capacitated spermatozoa. Both live and dead spermatozoa were labelled with oocyte plasma membrane, suggesting that oocyte plasma membrane binding sites detected by the flow cytometer are present on the surface of intact boar spermatozoa.

\section{Discussion}

In the present study, the capacitation-dependent and acrosome reaction-induced changes in oocyte plasma membrane binding sites on live boar spermatozoa were characterized using isolated oocyte plasma membrane. The washed spermatozoa ( $0 \mathrm{~h}$ incubated) have very 
limited ability to interact with the oocyte plasma membrane (Berger and Horton, 1988). Spermatozoa incubated for $6 \mathrm{~h}$ under these conditions (capacitated spermatozoa) have bound and subsequently fused with the pig oocyte plasma membrane (Linfor and Berger, 2000) and contributed to further embryonic development (M. Sween and T. Berger, unpublished).

The appearance of oocyte plasma membrane binding sites at the postacrosomal head and equatorial segment of live boar spermatozoa occurs with capacitation and induced acrosome reactions, indicating either an unmasking or redistribution of oocyte plasma membrane binding sites to these sperm surface subdomains. The capacitation-dependent increase in oocyte plasma membrane binding sites on the boar spermatozoa visualized by fluorescence microscopy was also demonstrated when the population distribution of OPM-Alexabound spermatozoa was analysed by flow cytometry. The percentage of spermatozoa with equatorial localization increased in the biologically relevant acrosome-reacting population. Modification of the sperm plasma membrane occurs with capacitation and acrosome reaction (Primakoff et al., 1987; Rochwerger and Cuasnicu, 1992; de Lamirande, 1997). Capacitation leads to changes in exposed sperm membrane proteins and antigen localization in boar spermatozoa (Berger, 1990; Topfer-Petersen, 1990; Peknicova et al., 1994). Control incubated populations confirmed that the changes in OPM-Alexa binding were capacitation-dependent as bicarbonate is necessary for capacitation (Harrison, 1996); bicarbonate is present in the medium because $\mathrm{CO}_{2}$ in the gas phase readily equilibrates with the medium and is rapidly converted to bicarbonate (Freshney, 2000).

The proportion of spermatozoa with detectable OPMAlexa binding was higher in the flow cytometric trials than in spermatozoa observed with fluorescence microscopy. This finding is probably due to the increased sensitivity of the flow cytometer. A reduction in the proportion of spermatozoa binding OPM-Alexa after induction of the acrosome reaction was detected by flow cytometry but not by microscopy, and this finding may be due to a loss of sites during the acrosome reaction.

With these data, the percentage of spermatozoa with the ability to interact with the oocyte plasma membrane after in vitro capacitation and after acrosome reaction induction after $6 \mathrm{~h}$ incubation in capacitating conditions can be determined. Capacitation of boar spermatozoa for $6 \mathrm{~h}$ allows for maximum sperm penetration of zona pellucida-free hamster oocytes and induced acrosome reaction rates are the same for spermatozoa incubated for $6 \mathrm{~h}$ and $4 \mathrm{~h}$ (Berger and Parker, 1989; Berger et al., 1989). This sperm population incubated for $6 \mathrm{~h}$ may not represent all the spermatozoa in the population that are capable of interacting with the oocyte plasma membrane. Sperm populations are heterogeneous presumably to prevent spermatozoa from all being competent for fertilization at the same time (Bedford, 1983; Amann

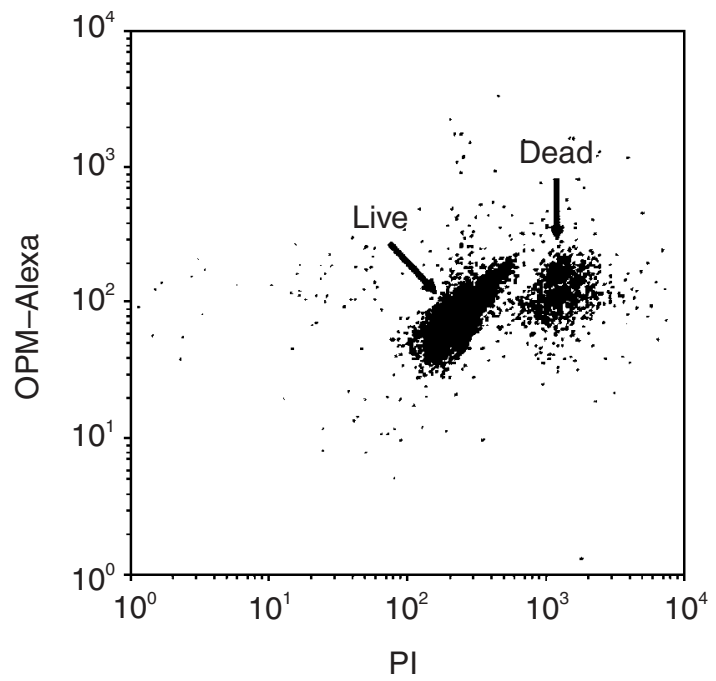

Fig. 5. Dotplot of flow cytometry analysis of OPM-Alexa labelling versus propidium iodide (PI) staining showing separation of live and dead boar sperm populations.

et al., 1993; Harrison 1997). The heterogeneity of sperm populations has been demonstrated in zona pellucida binding ability and capacitation (Harrison et al., 1993; Ashworth et al., 1995; Harkema et al., 1998).

In conclusion, a change in oocyte plasma membrane binding sites during capacitation and acrosome reaction of live boar spermatozoa has been demonstrated. Flow cytometric analysis of the oocyte plasma membrane binding sites confirmed an increase in binding sites with capacitation. Further studies with flow cytometric analysis of oocyte plasma membrane binding sites could be useful for studying the heterogeneous nature of the boar sperm population and fertility (Hammerstedt, 1996).

The authors thank B. Ball and G. Cherr for review of the manuscript. Grant support was from USDA NRICGP 99-02240 and W171RR (T. Berger) and Fertilization and Early Development Training Grant USPHS HD07131 (B. L. Sartini).

\section{References}

Ash K, Berger T, Munn RJ and Horner CM (1994) Isolation and partial purification of plasma membrane from porcine oocytes Molecular Reproduction and Development 38 334-337

Ashworth PJC, Harrison RAP, Miller NGA, Plummer JM and Watson PF (1995) Flow cytometric detection of bicarbonate-induced changes in lectin binding in boar and ram sperm populations Molecular Reproduction and Development 40 164-176

Amann RP, Hammerstedt RH and Veeramachaneni DNR (1993) The epididymis and sperm maturation: a perspective Reproduction, Fertility and Development 5 361-381

Bedford JM (1983) Significance of the need for sperm capacitation before fertilization in eutherian mammals Biology of Reproduction 28 108-120

Berger T (1990) Changes in exposed membrane proteins during in vitro capacitation of boar sperm Molecular Reproduction and Development 27 249-253

Berger T and Horton MB (1988) Evaluation of assay conditions for the zonafree hamster ova bioassay of boar sperm fertility Gamete Research 19 $101-111$ 
Berger T and Parker K (1989) Modification of the zona-free hamster ova bioassay of boar sperm fertility and correlation with in vivo fertility Gamete Research 22 385-397

Berger T, Turner KO, Meizel S and Hedrick JL (1989) Zona pellucidainduced acrosome reaction in boar sperm Biology of Reproduction $\mathbf{4 0}$ 525-530

de Lamirande E, Leclerc P and Gagnon C (1997) Capacitation as a regulatory event that primes spermatozoa for the acrosome reaction and fertilization Molecular Human Reproduction 3 175-194

Freshney RI (2000) Culture of Animal Cells Wiley-Liss, Inc., New York

Hammerstedt RH (1996) Evaluation of sperm quality: identification of the subfertile male and courses of action Animal Reproduction Science $\mathbf{4 2}$ $77-87$

Harkema W, Harrison RAP, Miller NGA, Topper EK and Woelders H (1998) Enhanced binding of zona pellucida proteins to the acrosomal region of intact boar spermatozoa in response to fertilizing conditions: a flow cytometric study Biology of Reproduction 58 421-430

Harrison RAP (1996) Capacitation mechanisms, and the role of capacitation as seen in eutherian mammals Reproduction, Fertility and Development 8 581-594

Harrison RAP (1997) Sperm plasma membrane characteristics and boar semen fertility Journal of Reproduction and Fertility Supplement $\mathbf{5 2}$ 195-211

Harrison RAP, Mairet B and Miller NGA (1993) Flow cytometric studies of bicarbonate-mediated $\mathrm{Ca}^{2+}$ influx in boar sperm populations Molecular Reproduction and Development 35 197-208

Koehler JK, DeCurtis I, Morton A, Stenchever MA, Smith D (1982) Interaction of human sperm with zona-free hamster eggs: a freezefracture study Gamete Research 6 371-386

Linfor J and Berger T (2000) Potential role of $\alpha v$ and $\beta 1$ integrins as oocyte adhesion molecules during fertilization in pigs Journal of Reproduction and Fertility 120 65-72
Peknicova J, Moos J, Mollova M, Srsen V and Capkova J (1994) Changes in immunochemical localisation of acrosomal and sperm proteins in boar spermatozoa during capacitation and induced acrosome reaction Animal Reproduction Science 35 255-271

Primakoff P, Hyatt H and Tredick-Kline J (1987) Identification and purification of a sperm surface protein with a potential role in sperm-egg membrane fusion Journal of Cell Biology 104 141-149

Rochwerger L and Cuasnicu PS (1992) Redistribution of a rat sperm epididymal glycoprotein after in vitro and in vivo capacitation Molecular Reproduction and Development 31 34-41

SAS Institute Inc. (1985) SAS ${ }^{\circledR}$ User's Guide: Statistics Version 5 SAS Institute Inc., Cary, NC

Talbot P and Chacon RS (1982) Ultrastructural observations on binding and membrane fusion between human sperm and zona-pelludica-free hamster oocytes Fertility and Sterility 37 240-248

Topfer-Petersen E, Friess AE, Stoffel M and Schill WB (1990) Boar sperm membrane antigens. II. Reorganization of an integral membrane antigen during capacitation and acrosome reaction Histochemistry 93491 495

Yanagimachi R (1994) Mammalian fertilization. In The Physiology of Reproduction pp 189-317 Eds E Knobil and JD Neill. Raven Press, New York

Young IT (1977) Proof without prejudice: use of the Kolmogorov-Smirnov test for the analysis of histograms from flow systems and other sources Journal of Histochemistry and Cytochemistry 25 935-941

Received 18 November 2002.

First decision 20 December 2002

Revised manuscript received 16 January 2003.

Accepted 3 March 2003. 